\title{
What can psychotherapy contribute to community psychiatry, and vice versa? - The North Devon experience
}

\author{
Jeremy Holmes, Consultant Psychiatrist, North Devon District Hospital, Barnstaple, \\ Devon EX31 4JB
}

This paper is based on experiences over the past three years in North Devon (population 140,000), a predominantly rural district that until 1986 based most of its psychiatric facilities in a large mental hospital in Exeter, nearly 40 miles away. When Exeter went 'Italian', services were devolved to local districts, and North Devon was given the responsibility for providing comprehensive psychiatric care based on a DGH unit, a Day Centre, two hostels and a small multidisciplinary team. No formal psychotherapy services were included in this plan. This is a familiar enough pattern, one that can be found throughout Europe and North America. I shall try to chart the movement from the heady days of decarceration to current uncertainties about community care, a state which might be called post-deinstitutionalism.

My account is informed by the distinction drawn by the sociologist Karl Mannheim (1936) between ideology and utopia. For Mannheim, ideology implies deception, albeit unconscious: a justification used by ruling groups to legitimise their position and practices. Utopia reflects the wish to escape from the dominance of the ruling group by those who lack power, based usually on a negation of the existing order. Both, for opposite reasons, avoid certain aspects of reality: ideology because it is static, utopia because of its insistence upon continuous change.

Community psychiatry has moved rapidly from utopia to ideology. In the days of the mental hospital, the hope of community-based psychiatry became a utopian ideal, a means of escape from the repressive and authoritarian structures of the total institution, an ideal society in which the mad were sacred and prejudice overcome. Now that community psychiatry has become the dominant paradigm it in turn is an ideology in which the problems of the postinstitutional era-the uncertain fate of the new chronic sick, interprofessional rivalries, fragmentation of services, transfer of resources away from mental illness - are concealed under the banner of normalisation. Utopian longings now take the form of a nostalgic wish to return to the good old days of the mental hospital where, like a mediaeval village in Merrie England, staff and patients lived harmoniously together. Alternatively a utopian dream of unlimited resources is envisioned.
Freud always insisted that the essence of neurosis was a turning away from reality. One task of psychoanalysis is to help people to face reality without the need to repress, deny or distort it. It should, however, be noted that psychotherapy itself is not immune from ideological or utopian aspects, when for example it denies the reality of mental illness or the need for drug therapy, or when it offers the false hope of discovering some primal trauma which, once identified, will lead to complete cure.

\section{The North Devon experience}

I shall now try to show how the introduction of a psychotherapeutic milieu in North Devon helped community psychiatry to move from ideology-based confusion to a more realistic and effective system of care.

Community psychiatry in North Devon started off in good heart, with an optimistic and enthusiastic team, housed in a spick and span new unit. But it was not long before difficulties became apparent. 'Nasty' patients were unwelcome as they wandered around the rest of the DGH; some had to be transferred back to neighbouring districts which had not progressed so fast towards mental hospital closure. Suicides occurred, and one patient died unexpectedly while in 'seclusion'. Confusion and disillusionment began to surface.

The problems experienced by the staff manifested themselves in three main ways: splitting, envy, and delinquency. Splits were legion. One consultant was seen as 'bad', another 'good'; one ward was seen as having 'nice' patients, another as having all the difficult ones. There was little respect between the inpatient unit and the day centre. Tension and rivalry existed between community workers whose values were vaguely psychotherapeutic or systems-based, and the medical 'pill-pushers'.

At times of crisis, especially with difficult patients, these splits became chasms. When a patient committed suicide the consultants blamed the junior staff, or each other. On Ward X the staff complained about Consultant A's patients who stayed for 'ever' and 'never' got better, unlike Consultant B's; on Ward Y it was the reverse. The community team complained 
that they could 'never' get hold of the hospital team when they needed to, and vice versa.

Envy was also widespread. The surgeons who had been pressing their claim for new operating theatres for years were appalled to see the opening of a brand new psychiatric block, and there was a prevalent myth that psychiatry had somehow hi-jacked local funds rather than, as was in fact the case, benefiting from monies transferred from the closure of the mental hospital in the nearby district. Some GPs were dissatisfied with the service and one was heard to complain, echoing Williams \& Clare (1981) that, compared with ten years ago, there were now twice as many psychiatrists seeing half the number of patients.

Perhaps the most serious problem was a subtle delinquency among a few staff members. The majority were devoted hard-working professionals but these exceptions easily became scapegoats and seemed to reflect a dysfunctional aspect of the whole unit. The delinquency took many forms, from minor examples such as never coming to staff meetings, to the more serious such as drinking while on duty, or unavailability when on call.

This was by no means the whole picture. As with a borderline patient, the unit contained areas of normal or even good functioning which co-existed with the problems described. Many of the difficulties were seen as intractable or insoluble and a blind eye was turned to them. Nevertheless, there was a general just-below-the-surface awareness of the atmosphere depicted that led to a discomfort and disillusionment about community psychiatry. Nor was I exempt from these difficulties, being often inwardly and sometimes openly censorious of what passed for 'therapy' among some staff members who flagrantly transgressed boundaries by treating patients at irregular hours in their homes, even at times meeting them for a drink in the pub! What passed for 'psychotherapy' was often superficial and half-baked, with frequent 'frame-violations' (Langs, 1986) leading to unconscious role reversal between client and therapist. No doubt these criticisms were valid, but a lot of good supportive work was undervalued by such dismissal, and they added to the general atmosphere of splitting and mistrust, and were partly defensive, since my psychoanalytic orientation seemed less immediately appealing than common-sense counselling and behavioural methods or cathartic sessions of emotional release.

\section{The contribution of psychotherapy to community psychiatry}

Like community psychiatry, psychotherapy also has an ideology in the triple sense of a set of working models, ideals to be striven for, and, at times, a way of avoiding certain aspects of reality. The values of psychotherapy (Holmes \& Lindley, 1989) include:

an emphasis on fostering autonomy so that patients are no longer in thrall to their illness or difficulties, but can choose to direct their life as they would wish. Unlike community psychiatry, which emphasises external barriers to autonomy, psychotherapy aims to remove internal obstacles so helping the patient to feel more free in relation to himself;

the view that a prerequisite for the development of autonomy is a secure setting in which the patient feels held and contained;

a holistic approach that takes in the entire field. Psychoanalytically this refers to unconscious and irrational elements, including those of the staff; from a systemic perspective it means that patients, the institution, and the staff have to be considered as a system in which no one element is privileged; an emphasis on thought rather than action, and on the need to create a space for reflection before change can occur;

a developmental perspective that recognises the need for differentiation and acknowledges real differences between people, based ultimately on Chasseguet-Smirgel's (1985) 'double difference': the difference between the sexes and the difference between the generations.

It is worth noting at this point that there has been an 'ecological' (Malan, 1963) shift in psychotherapy, mirroring the movement in psychiatry from institution to community. Psychotherapists are increasingly preoccupied with containment and holding as they face problems of splitting and fragmentation, rather than, as in the past, focusing on repression and the need to overcome authoritarian structures, both internal and external.

There are several important implications of this perspective for community psychiatry. The mental hospital provided not just a physical structure but also a 'second skin' (Bick, 1988) for patients and staff, analogous to the containing function provided by the mother and the family which is then internalised as the child develops. Community psychiatry finds it physically hard to contain its difficult and disturbed patients. Equally important is the lack of the psychological containment needed to hold together a unit divided into numbers of smaller subunits that communicate badly or not at all with one another, serving patients whose inner and outer worlds are also characterised by fragmentation and splitting.

Second, the holistic psychotherapeutic approach recognises the inevitability of negative feelings towards the mentally ill, both in society at large and in the form of counter-transference reactions among staff. It is confusing for administrators or psychiatric 
workers who, following the ideals of community psychiatry, champion the 'rights' of the mentally ill to be treated 'just like any other ill patients', to find they meet with hostility in the public and, even more disturbingly, within themselves.

Another source of confusion within community psychiatry derives from the breakdown of the traditional medical hierarchy when patients are treated in the community. There is a movement from role rigidity to role blurring within the small units; at the same time community nurses, occupational therapists, social workers, psychologists and others wish to work as independent practitioners rather than submit to psychiatric hegemony. Mollon (1989) has written of the 'narcissistic perils' which befall practitioners when faced with difficult and disturbed patients armed only with an 'all-you-need-is-love' model that de-emphasises pathology, dismisses the idea that the inner world of the patient may be damaged or distorted, and sees only 'environmental failure', 'problems in living' or 'behavioural difficulties'. Workers easily become confused and discouraged and begin to lose faith in themselves and start to take avoiding action rather than face problems.

With its developmental perspective and emphasis on differentiation, psychotherapy can help clarify and sometimes resolve some of these difficulties by offering a model of pathology that takes account of the internal world and at the same time is not dismissible as a medical 'label'. It can also recognise real differences between practitioners in ability, experience and training without simply reinforcing existing hierarchies, thereby providing a path between the 'what I say goes' of the traditional model and the 'anything goes' of community confusion.

\section{The preconditions for a psychotherapy service}

A psychotherapy service can make three main contributions to the work of a psychiatric unit (Margison et al, 1989). First it is a primary treatment for a number of important conditions including mildmoderate depression, some personality disorders, eating disorders and post-traumatic stress disorder. Second it is an adjuvant to other methods of treatment as in the use of family therapy in schizophrenia. Third it has a role in the support, supervision and training of staff. The main emphasis in this account however is on the preconditions for the setting up of such a service. It is necessary to create a culture that is sympathetic and receptive to psychotherapy before a service can be effective, just as, especially with unsophisticated patients, psychotherapy outcome is improved if treatment is preceded by explanatory sessions (Beutler et al, 1986). Setting up a psycho- therapy service within a culture that is rife with splitting and envy can merely accentuate those tendencies and runs the risk of being marginalised, or seen as an inessential 'luxury' (Holmes \& Lindley, 1989).

The first objective in North Devon then was not necessarily to start 'doing psychotherapy', but to create a safe containing setting, based on mutual respect, which at the same time recognised real differences between workers. Two practical steps were taken to this end. First two adult psychotherapist posts were created. One was only two sessions per week, but setting up this post and the discussions which this entailed, especially with managers, had an important role in educating and explaining the nature and necessity for psychotherapists as part of the community psychiatric team.

The second step was the establishment of a psychotherapy centre and a half-day psychotherapy training seminar. These evolved from an analysis of the work of the day centre which showed that it provided a sometimes muddled mixture of drop-in facility, day care and sessional work. The centre was re-organised so that day care continued for three days per week but on the other two the centre was used for psychotherapy, providing both a service, and through the seminar, staff education and supervision.

A striking feature of contemporary community psychiatry is that, perforce, 'we are all psychotherapists now'. Most mental health workers CPNs, psychologists, OTs, junior psychiatrists - are doing psychotherapeutic work as best they can, often with difficult clients, largely untutored and unsupervised, often with very limited training in psychotherapy. The aim of the seminar is to provide a forum for a multidisciplinary group of community workers in which education and supervision are at least equal in intensity and continuity to the therapeutic requirements of their clients. The seminar offers a model of a secure and regular setting within which anxieties can be expressed and learning can take place. It has also led to increased mutual knowledge and respect between the different psychotherapy disciplines. The analytic therapists have learned about cognitive therapy, and no longer see it as a palliative which ignores deep issues, but as a powerful form of brief therapy. Cognitive therapists no longer view analytic approaches as woolly and interminable, but as the treatment of choice for patients with personality difficulties and long-standing relationship difficulties. On the basis of this, and other rapprochements it has been possible to establish an integrated psychotherapy service offering a range of therapies, including family therapy, cognitive therapy, analytic therapy and a number of short and long term groups.

There have also been a number of external consequences of the group. Some staff members have left, making way for others more in tune with the 
prevailing ideology. There is a growing interest in formal psychotherapy training (which the seminar does not aim to replace) and several members have either completed or are undertaking such training in analytic therapy, cognitive therapy, neurolinguistic programming and family therapy. A third development has been the recognition of the need for psychotherapeutic work in the rest of the unit, and one of the psychotherapists now runs a staff support group at the DGH unit, which has led to improved morale and reduced splitting among the staff there.

\section{What can psychotherapy learn from community psychiatry?}

So far community psychiatry has been depicted as a frail damsel menaced by the dragons of role blurring and good intentions, saved by the shining knight of psychotherapy. It must be emphasised that the benefits are by no means all one way. Just as community psychiatry needs saving from therapeutic promiscuity, so psychotherapy, like Rapunzel, needs to be released from imprisonment in its ivory tower, to let down its hair a little. The principle that services need to be available to a whole population, not just to a particular section who have the ability to pay, the necessary intelligence, a suitable illness or personality, has not yet been fully accepted within psychotherapy. Community psychiatry sees the need for a network of graduated facilities and levels of intervention if the whole range of difficulties and illnesses are to be met (Bennett, 1978). Psychotherapy needs to learn from this 'flexible response' and to offer a range of interventions appropriate to the differing psychotherapeutic needs of a whole population.

This pluralistic approach will mean much greater mutual respect between the different psychotherapeutic schools if psychotherapy is to offer a client/ treatment matrix of the kind envisaged by Paul (1967) when he asked what treatment, by whom, is most effective for this individual, with what specific problem, and under which set of circumstances.

\section{Comment}

This paper has tried to show how psychotherapy and community psychiatry may mutually benefit one another. Only with a strong psychotherapy presence within the NHS will this be possible. Haunting both disciplines is the issue of evaluation. The basis of psychotherapy is the creation of meanings out of confusion, wholeness out of fragmentation. Health workers are now being asked to put meanings not into words but figures. If a community psychotherapy service is to become a reality rather than a utopian ideal, meeting that challenge must become an urgent task.

\section{References}

BenNett, D. H. (1978) Community psychiatry. British Journal of Psychiatry, 132, 209-220.

Beutler, L. E., Crago, M. \& Arizmendi, T. G. (1986) Process and outcome in psychotherapy. In Handbook of Psychotherapy and Behaviour Change (eds. S. L. Garfield and A. E. Bergin). Chichester: Wiley.

Bick, E. (1988) The experience of the skin in early object relations. In Melanie Klein Today Volume I. London: Routledge.

Chasseguet-Smirgel, J. (1985) Creativity and Perversion. London: Free Association Books.

Holmes, J. \& Lindley, R. (1989) The Values of Psychotherapy. Oxford: Oxford University Press.

Langs, R. (1986) A Primer of Psychotherapy. New York: Croom Helm.

Malan, D. (1973) A Study of Brief Psychotherapy. London: Tavistock.

MANNHEIM, K. (1936) Ideology and Utopia (trans. L. Wirth and E. Shils). London: Routledge.

Margison, F., Grant, S. \& Powell, A. (1989) Psychotherapy services in the NHS. Document prepared for the Royal College of Psychiatrists Psychotherapy Specialist Section.

Mollon, P. (1989) Anxiety, supervision and a space for thinking: some narcissistic perils for clinical psychologists learning psychotherapy. British Journal of Medical Psychology, 62, 113-122.

PAul, G. L. (1967) Outcome research in psychotherapy. Journal of Consulting Psychology, 31, 109-118.

Williams, P. \& Clare, A. (1981) Changing patterns of psychiatric care. British Medical Journal, 282, 375-377. 